\title{
Momentum-Space Atom Correlations in a Mott Insulator
}

\author{
Cécile Carcy, Hugo Cayla, Antoine Tenart, Alain Aspect, Marco Mancini, and David Clément $\odot^{*}$ \\ Laboratoire Charles Fabry, Institut d'Optique Graduate School, CNRS, Université Paris Saclay, \\ 2 Avenue Augustin Fresnel, 91127 Palaiseau cedex, France
}

(Received 24 April 2019; published 7 November 2019)

\begin{abstract}
We report on the investigation of the three-dimensional single-atom-resolved distributions of bosonic Mott insulators in momentum space. First, we measure the two-body and three-body correlations deep in the Mott regime, finding a perfectly contrasted bunching whose periodicity reproduces the reciprocal lattice. In addition, we show that the two-body correlation length is inversely proportional to the in-trap size of the Mott state with a prefactor in agreement with the prediction for an incoherent state occupying a uniformly filled lattice. Our findings indicate that the momentum-space correlations of a Mott insulator at small tunneling are those of a many-body ground state with Gaussian statistics. Second, in the Mott insulating regime with increasing tunneling, we extract the spectral weight of the quasiparticles from the momentum-density profiles. On approaching the transition towards a superfluid, the momentum spread of the spectral weight is found to decrease as a result of the increased mobility of the quasiparticles. While the shapes of the observed spectral weight agree with those predicted by perturbative many-body calculations for homogeneous systems, the fitted mobilities are larger than the theoretical ones, mostly because of the coexistence of various phases in the trap.
\end{abstract}

DOI: 10.1103/PhysRevX.9.041028

Subject Areas: Atomic and Molecular Physics

\section{INTRODUCTION}

Measuring many-body correlations is central to investigate and reveal the properties of strongly interacting quantum matter [1]. It allows one to test the predictions of simple microscopic models used to understand manybody physics [2]. One paradigmatic example is the Hubbard Hamiltonian that describes quantum particles hopping between the sites of a lattice in the presence of interaction. A comparison of the predictions of the Hubbard model with the measured response functions, e.g., the one-particle spectral function [3] and the dynamical structure factor [4], shows its relevance for a large variety of systems, from transition-metal oxides [5] and heavy fermions [6] to high$T_{c}$ superconductors [7]. A direct probe of correlations between individual particles is yet hardly possible to implement in solids. Thanks to single-atom-resolved detection methods [8], quantum gases offer an alternative test bed for many-body theories.

The celebrated Mott transition, a generic metal-toinsulator transition of Hubbard models [9], is investigated

\footnotetext{
* Corresponding author. david.clement@institutoptique.fr

Published by the American Physical Society under the terms of the Creative Commons Attribution 4.0 International license. Further distribution of this work must maintain attribution to the author(s) and the published article's title, journal citation, and DOI.
}

with quantum gas microscopes, illustrating the benefits from probing many-body physics at the single-particle level. Position-space two-particle correlations in Mott insulators indeed yield unprecedented and quantitative observations, such as the reduction of on-site atom fluctuations $[10,11]$, string order in low dimensions [12], outof-equilibrium dynamics [13,14], and antiferromagnetic ordering $[15,16]$. On the other hand, the investigation of momentum-space correlations in atomic Mott states are scarce, and, in particular, there has been no implementation of a single-atom-resolved probe of momentum correlations. Momentum-resolved light-scattering techniques are implemented to measure spectral functions [17-19], and the noise correlation analysis of time-of-flight (TOF) absorption images [20] reveals density correlations in expanding Mott insulators [21,22]. But these probes do not permit quantitative studies of correlation functions, including their shape and their width, due to limited resolution in momentum, line-of-sight integration, and lack of singleparticle sensitivity. Experimental signatures of the contribution of particle-hole excitations to the coherence of a Mott insulator are extracted from the visibility [23] and the density modulation of TOF distributions [24]. But a momentum-resolved measure of this contribution when approaching the Mott transition, i.e., an observation of the modifications of the spectral weight of the quasiparticles in momentum space, was not possible. Recently, however, it has become possible to measure momentum-space correlations, in either one- or two-dimensional geometries using 
optical imaging [25-27] or in three dimensions with the species metastable helium ( $\left.\mathrm{He}^{*}\right)$, which can be detected at the single-atom level $[28,29]$.

In this work, we report on using momentum-space single-atom-resolved distributions [29] in $\mathrm{He}^{*}$ gaseous Mott insulators to investigate both the average population of the momentum states (i.e., the momentum density) and its fluctuations (through two-body and three-body momentum correlations). Deep in the Mott regime, we observe a perfectly contrasted momentum bunching, with secondand third-order correlation functions whose structure reproduces the reciprocal lattice. Moreover, from the shape of the bunching peaks, we determine the two-particle correlation length in Mott insulators of various in-trap sizes, finding an excellent agreement with $a b$ initio calculations. These features show that in the limit of vanishing tunneling the Mott correlations are driven by a Gaussian density operator for bosons uniformly distributed in the lattice sites. From cuts in the three-dimensional (3D) momentum density, we also extract the spectral weight $Z(k)$ of the Mott quasiparticles. The evolution of $Z(k)$ when the system approaches the superfluid-to-Mott transition provides a direct signature of the increased spatial coherence of the quasiparticles. At the quantitative level, however, a comparison with perturbative many-body approaches reveals discrepancies which we discuss.

\section{MOMENTUM-SPACE CORRELATION FUNCTIONS OF A MOTT STATE: THEORY IN A NUTSHELL}

A Mott insulator is an insulating phase caused by the presence of strong interactions between particles moving in crystalline structures. Hubbard models provide a microscopic description of the Mott physics based on the interplay between interaction and kinetic energy. When the ratio $U / J$ of the on-site (repulsive) interaction energy $U$ to the hopping energy $J$ between adjacent lattice sites increases, the ground state of a Hubbard Hamiltonian undergoes a phase transition from a conductor to a Mott insulator. The Fermi Hubbard model is central for the understanding of metal-insulator transitions in solids when electron-electron interactions are important [5,9]. Recently, quantum gases have permitted the observation of a Mott transition with bosons [30], which is described by the BoseHubbard Hamiltonian [31]

$$
H=-J \sum_{\left\langle l, l^{\prime}\right\rangle} \hat{b}_{l}^{\dagger} \hat{b}_{l^{\prime}}+\frac{U}{2} \sum_{l=1}^{N_{\text {site }}} \hat{n}_{l}\left(\hat{n}_{l}-1\right),
$$

where $\hat{b}_{l}$ is the annihilation operator of a particle on site labeled $l$ and $\hat{n}_{l}=\hat{b}_{l}^{\dagger} \hat{b}_{l}$. The critical value for the Mott transition in the 3D Bose-Hubbard Hamiltonian is calculated numerically and found equal to $(U / J)_{c}=29.3$ [32]. The properties of the Mott state- such as the amplitude of the gap in the excitation spectrum or the role of particlehole excitations - change when varying $U / J$ from deep in the insulating regime $\left[U / J \gg(U / J)_{c}\right]$ to the value at the phase transition. In a first approximation, these modifications can be viewed as resulting from the contribution of some particle and hole excitations on top of a uniformly filled lattice. In quantum gas experiments, the ratio $U / J$ can be varied continuously by changing the amplitude of the lattice potential, allowing one to investigate the modifications of that many-body ground state (the Mott insulator) with a high degree of control [30]. In the following, we describe the properties of the Mott state in the momentum space. To this aim, we introduce the momentum-space operators

$$
\hat{a}(\vec{k})=\frac{1}{\sqrt{V}} \sum_{l=1}^{N_{\text {site }}} e^{i \vec{k} \cdot \vec{r}_{l}} \hat{b}_{l},
$$

where the volume of quantization $V$ is chosen as the in-trap volume of the gas. As a consequence of the crystalline structure of the lattice, all momentum-space quantities are periodic with the period of the reciprocal lattice $k_{d}=2 \pi / d$, where $d$ is the lattice spacing, e.g., $\hat{a}(\vec{k}+\vec{K})=$ $\hat{a}(\vec{k})$ with $\vec{K}=k_{d}\left(n_{x} \vec{u}_{x}+n_{y} \vec{u}_{y}+n_{z} \vec{u}_{z}\right)$, where $\left\{\vec{u}_{j}\right\}_{x, y, z}$ are the orthonormal vectors associated to the lattice and $\left\{n_{j}\right\}_{x, y, z}$ are integers.

\section{A. Many-body correlations in a perfect Mott insulator}

Deep in the Mott phase at a large amplitude of the lattice potential, the tunneling becomes vanishingly small, and it is standard to approximate the many-body ground state by a "perfect" Mott insulator, i.e., a Mott insulator with no coupling between the sites, $J=0$. The perfect Mott insulator for the Hamiltonian of Eq. (1) with unity occupation of the lattice site is then $|\psi\rangle_{J=0}=\Pi_{l} \hat{b}_{l}^{\dagger}|0\rangle$. The absence of phase coherence between the lattice sites, $\left\langle\psi\left|\hat{b}_{l}^{\dagger} \hat{b}_{l^{\prime}}\right| \psi\right\rangle_{J=0}=\left\langle\hat{b}_{l}^{\dagger} \hat{b}_{l^{\prime}}\right\rangle_{J=0}=\delta_{l, l^{\prime}}$, has important consequences on the momentum-space properties. Under these conditions, the correlations between two momentum operators [see Eq. (2)] take the form

$$
\left\langle\hat{a}^{\dagger}(\vec{k}) \hat{a}\left(\overrightarrow{k^{\prime}}\right)\right\rangle_{J=0}=\frac{1}{V} \sum_{l=1}^{N_{\text {site }}}\left\langle\hat{n}_{l}\right\rangle e^{i\left(\overrightarrow{k^{\prime}}-\vec{k}\right) \cdot \vec{r}_{l}} .
$$

The momentum density of a perfect Mott insulator is thus constant: $\rho(\vec{k})=\left\langle\hat{a}^{\dagger}(\vec{k}) \hat{a}(\vec{k})\right\rangle_{J=0}=\rho(\overrightarrow{0})$. In addition, the correlations between momentum components separated by more than the inverse of the system size $L$ are vanishingly small. On the contrary, the sum in Eq. (3) is nonzero for $\left|\vec{k}-\overrightarrow{k^{\prime}}\right|<2 \pi / L$, which implies that some correlations are present in momentum space even if there are no positionspace correlations, a situation similar to that described by 
the van Cittert-Zernike theorem in optics [33]. Equation (3) defines the one-particle volume of coherence, i.e., the volume over which the first-order correlation function $g^{(1)}\left(\vec{k}, \vec{k}^{\prime}\right)=\left\langle\hat{a}^{\dagger}(\vec{k}) \hat{a}\left(\overrightarrow{k^{\prime}}\right)\right\rangle / \sqrt{\rho(\vec{k}) \rho\left(\vec{k}^{\prime}\right)}$ is nonzero. This momentum-space volume of coherence reflects the distribution of atoms $\left\{\left\langle\hat{n}_{l}\right\rangle\right\}_{l}$ in the lattice.

As pointed out in Ref. [20], higher-order momentumspace correlations can reveal some properties of many-body ground states even when the momentum density is featureless. For instance, bosonic bunching is expected in the two-body correlations $g^{(2)}\left(\vec{k}, \overrightarrow{k^{\prime}}\right)=$ $\left\langle\hat{a}^{\dagger}(\vec{k}) \hat{a}^{\dagger}\left(\overrightarrow{k^{\prime}}\right) \hat{a}(\vec{k}) \hat{a}\left(\overrightarrow{k^{\prime}}\right)\right\rangle / \rho(\vec{k}) \rho\left(\overrightarrow{k^{\prime}}\right)$ associated with finding one particle with a momentum $\vec{k}$ and a second one with a momentum $\overrightarrow{k^{\prime}}$. In the case of the perfect Mott state, that is to say, when the tunneling is zero, the amplitude and the width of the bunching effect can be predicted accurately. Indeed, the atom number per lattice site is then fixed. For a unity occupation of the lattice $n_{l}=1$, the Hamiltonian of Eq. (1) reduces to that of noninteracting particles and is diagonal in the momentum-space basis. As a result, the many-body momentum-space correlations are those of uncorrelated bosons with a Gaussian density operator [34]. For such a Gaussian many-body ground state, the Wick decomposition yields $g^{(2)}\left(\vec{k}, \vec{k}^{\prime}\right)=1+\left|g^{(1)}\left(\vec{k}, \vec{k}^{\prime}\right)\right|^{2}$. In particular, the amplitude of the two-body correlation at zero particle distance is twice that found for uncorrelated particles: $g^{(2)}(\vec{k}, \vec{k})=2$. In addition, the shape of the bunching peak provides quantitative information about the in-trap profile. Its exact shape is set by the term $\left|g^{(1)}\left(\vec{k}, \vec{k}^{\prime}\right)\right|^{2}$, whose size determines the volume of coherence. Interestingly, higherorder correlation functions are also related to the first-order correlation function $g^{(1)}\left(\vec{k}, \overrightarrow{k^{\prime}}\right)$ when the Wick theorem applies (see Ref. [35], for instance).

\section{B. Momentum density and spectral weight of a Mott insulator at finite tunneling}

At finite tunneling $J>0$, a more elaborate picture than that of the perfect Mott state $|\psi\rangle_{J=0}$ must be introduced to account for the kinetic energy term in the Bose-Hubbard Hamiltonian. To do so, various many-body treatments introduce quadratic quantum fluctuations on top of the classical Gutzwiller solution for the perfect Mott [36-38]. In these approaches, the low-energy excitations of the Mott state are quasiparticles consisting of the combination of a doublon and a hole. At finite $J$, the many-body ground state contains a finite fraction of these particle-hole excitations. In addition, the mobility of the quasiparticles in the lattice restores some phase coherence. These modifications of the many-body ground state are described by the formalism of the Green function [2]. For our purposes, the Fourier transform of the Green function, i.e., the one-particle spectral function $\mathcal{A}(\vec{k}, \omega)$, is of special interest, as it is linked to the momentum density $\rho(\vec{k})$ of the Mott insulator [38]: $\rho(\vec{k}) \propto-\int_{-\infty}^{0} d \omega \mathcal{A}(\vec{k}, \omega)$. Replacing $\mathcal{A}(\vec{k}, \omega)$ by its expression, one finds that the momentum density $\rho(\vec{k})$ of the Mott insulator is directly related to $Z(\vec{k})$, the spectral weight of the quasiparticles:

$$
\rho(\vec{k})=\mathcal{N}[Z(\vec{k})-1],
$$

where $\mathcal{N}$ is a normalization factor. The spectral weight quantifies the overlap between the many-body wave function with one added particle (or hole) and the true excited one-particle (or one-hole) state. In the noninteracting case, $Z(\vec{k})$ is a delta function, because a state with one more particle or hole is still an eigenstate of the system. In the correlated case, many momentum eigenstates have a nonzero overlap with the state formed by simply adding a particle or a hole. As a consequence, the spectral weight acquires a finite momentum support whose shape reflects these correlations.

The momentum density $\rho(\vec{k})$ of the Mott state thus provides a direct probe of its spectral weight $Z(\vec{k})$. The spectral weight of a homogeneous Mott with unity occupation of the lattice sites can be calculated using perturbative many-body approaches [38-40]. Deep in the Mott regime $(U / J \gg 1), Z(k)$ is a small-amplitude oscillating function of period $k_{d}=2 \pi / d$, with $d$ the lattice spacing $[38,40,41]$. These oscillations stem from the presence of a small number of quasiparticles that hop between adjacent lattice sites only. On approaching the Mott transition, the spectral weight is expected to become increasingly peaked around the reciprocal lattice vectors $\vec{K}$, as an added particle (or hole) can tunnel over several lattice sites [38,40,41].

\section{EXPERIMENTAL RESULTS}

\section{A. Observation of the Mott transition with metastable helium-4 atoms}

The experimental sequence starts with the production of a Bose-Einstein condensate (BEC) of metastable helium-4 atoms $\left({ }^{4} \mathrm{He}^{*}\right)$ in a crossed optical trap as described in Ref. [42]. The BEC is then loaded into the lowest-energy band of a 3D cubic optical lattice with a spacing $d=$ $775 \mathrm{~nm}$ and an amplitude $V_{0}=s E_{r}$, with $E_{r}=h^{2} / 8 m d^{2}$ the recoil energy [29]. The lattice amplitude $s$ is increased linearly at a rate of $0.3 \mathrm{~ms}^{-1}$, while, simultaneously, the intensity of the optical trap is switched off with a linear ramp of duration $20 \mathrm{~ms}$. The residual harmonic potential due to the Gaussian intensity profiles of the orthogonal lattice beams is isotropic with a trapping frequency $140 \sqrt{s} \mathrm{~Hz}$. We calibrate the amplitude $s$ by performing amplitude modulation spectroscopy, and we numerically calculate $J$ and $U$ from the value $s$. The rms uncertainty on $U / J$ is estimated to be $5 \%$. 
(a)

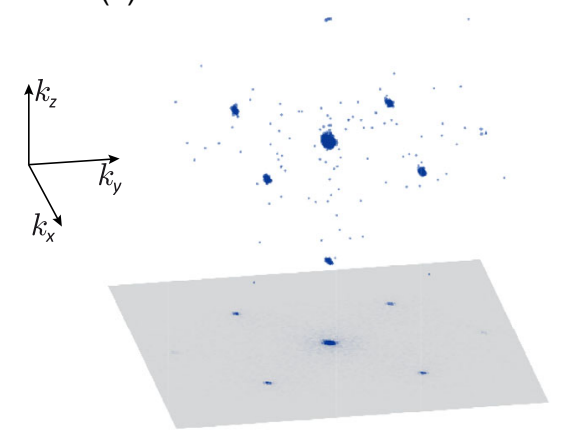

(b)

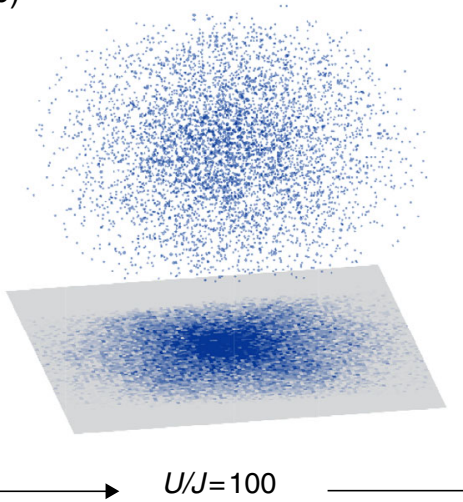

(c)

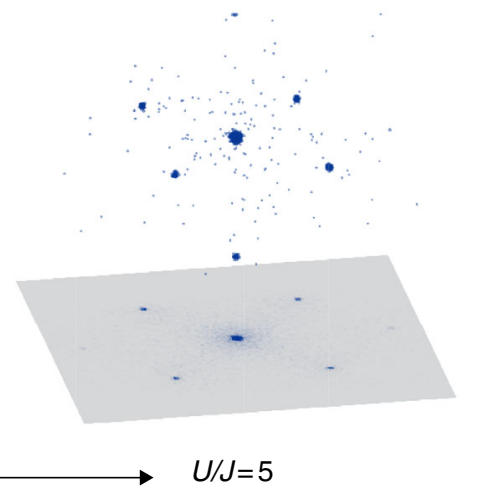

FIG. 1. Observation of the superfluid-to-Mott transition in momentum space with ${ }^{4} \mathrm{He}^{*}$ atoms. Far-field three-dimensional atom distributions, along with the corresponding two-dimensional projections in the $\left(k_{x}, k_{y}\right)$ plane. Each blue dot is an individual atom revealed by the $\mathrm{He}^{*}$ detector. (a) Superfluid regime at $U / J=5$, (b) Mott regime $U / J=100$, (c) superfluid regime $U / J=5$ restored when sweeping back from $U / J=100$ to $U / J=5$. With this set of data, the visibility of the interference pattern [23] goes from $\mathcal{V}=0.96$ in (a) to $\mathcal{V}=0.92$ in (c). This observation demonstrates that the loading ramps are adiabatic with a good approximation.

In the experiment, we measure time-of-flight singleatom-resolved distributions in the far field, from which we can calculate the 3D momentum density as well as higherorder correlation between individual atoms. We probe the gas after a time of flight of $t_{\text {tof }}=297 \mathrm{~ms}$ with the time- and space-resolved He* detector described in Ref. [43]. The $\mathrm{He}^{*}$ detector reveals the $3 \mathrm{D}$ positions $\vec{r}_{\text {tof }}$ of individual ${ }^{4} \mathrm{He}^{*}$ atoms in the center-of-mass reference frame with an excellent resolution (see the Appendix B). Importantly, $t_{\text {tof }}$ is larger than the time required to enter the far-field regime of propagation $t_{\mathrm{FF}}=m L^{2} / 2 \hbar \sim 30 \mathrm{~ms}$ for our experimental parameters, where $L \sim 40 d$ is the in-trap total size of the gas. In addition, interaction can be neglected during the expansion, as the TOF dynamics is driven by the large trapping frequency $(\gtrsim 100 \mathrm{kHz})$ of the individual lattice sites [44]. Indeed, in a previous work [29], we validate quantitatively the assumption of ballistic expansion from the lattice, i.e., $\hbar \vec{k}=m \vec{r}_{\text {tof }} / t_{\text {tof }}$, where $\vec{k}$ is the momentum of the atom in the lattice and $\vec{r}_{\text {tof }}$ is the measured atom position after a time of flight $t_{\text {tof }}$. With the ballistic assumption, the far-field TOF density $\rho_{\infty}\left(\vec{r}_{\text {tof }}, t_{\text {tof }}\right)$ yields the in-trap momentum density $\rho(\vec{k})$ :

$$
\rho(\vec{k})=\frac{1}{|\tilde{\omega}(\vec{k})|^{2}}\left(\frac{\hbar t_{\text {tof }}}{m}\right)^{3} \rho_{\infty}\left(\vec{r}_{\text {tof }}, t_{\text {tof }}\right),
$$

where $\tilde{\omega}(\vec{k})$ is the Fourier transform of the Wannier function in each lattice site. We determine $\tilde{\omega}(\vec{k})$ numerically in 3D and rescale the measured density $\rho_{\infty}\left(\vec{r}_{\text {tof }}, t_{\text {tof }}\right)$ according to Eq. (5). Doing so, $\rho(\vec{k})$ can be directly compared to the momentum density of the discrete Bose-Hubbard Hamiltonian introduced in the theory section [see Eq. (1)].

In Fig. 1, we plot examples of 3D TOF distributions across the Mott transition. The Mott transition has been investigated with various atomic species [45], and here we report its observation with metastable helium- 4 atoms, which allows us to obtain 3D single-atom-resolved distributions. Each blue dot in Fig. 1 corresponds to one detected atom. In Fig. 1(a), the distribution displays sharp diffraction peaks located at $k_{d}=2 \pi / d$, a manifestation of the long-range coherence of the superfluid state at $U / J=5$. Deep in the Mott insulating regime $(U / J=100)$, the phase coherence is lost [see Fig. 1(b)]. By decreasing $U / J$ from the Mott state back to the superfluid regime, the long-range coherence is restored, as illustrated by the high visibility of the diffraction peaks in Fig. 1(c). In the following, we focus on the Mott insulator regime using values of the ratio $U / J$ larger than the critical value for the Mott transition $(U / J)_{c}=29.3[32]$.

\section{B. Many-body momentum-space correlations deep in the Mott insulating regime}

We first discuss the single-atom-resolved distributions measured deep in the Mott insulator regime. As expected, we find that the momentum density $\rho(k)$ [as defined in Eq. (5)] is almost constant in this regime. To characterize the fluctuations of the momentum-space population, we introduce the two-particle correlation function defined as (see Appendix C for details)

$$
g^{(2)}(\delta \vec{k})=\frac{\int d \vec{k}\left\langle\hat{a}^{\dagger}(\vec{k}) \hat{a}^{\dagger}(\vec{k}+\delta \vec{k}) \hat{a}(\vec{k}+\delta \vec{k}) \hat{a}(\vec{k})\right\rangle}{\int d \vec{k} \rho(\vec{k}) \rho(\vec{k}+\delta \vec{k})},
$$

where $\hat{a}(\vec{k})$ is the annihilation operator associated to finding a particle at position $\vec{k}$ with the $\mathrm{He}^{*}$ detector. To facilitate the presentation and the discussion of the twobody correlation function, we plot $g^{(2)}(\delta \vec{k})$ along some specific directions $\vec{u}$ that can be chosen at will. In Fig. 2, we 

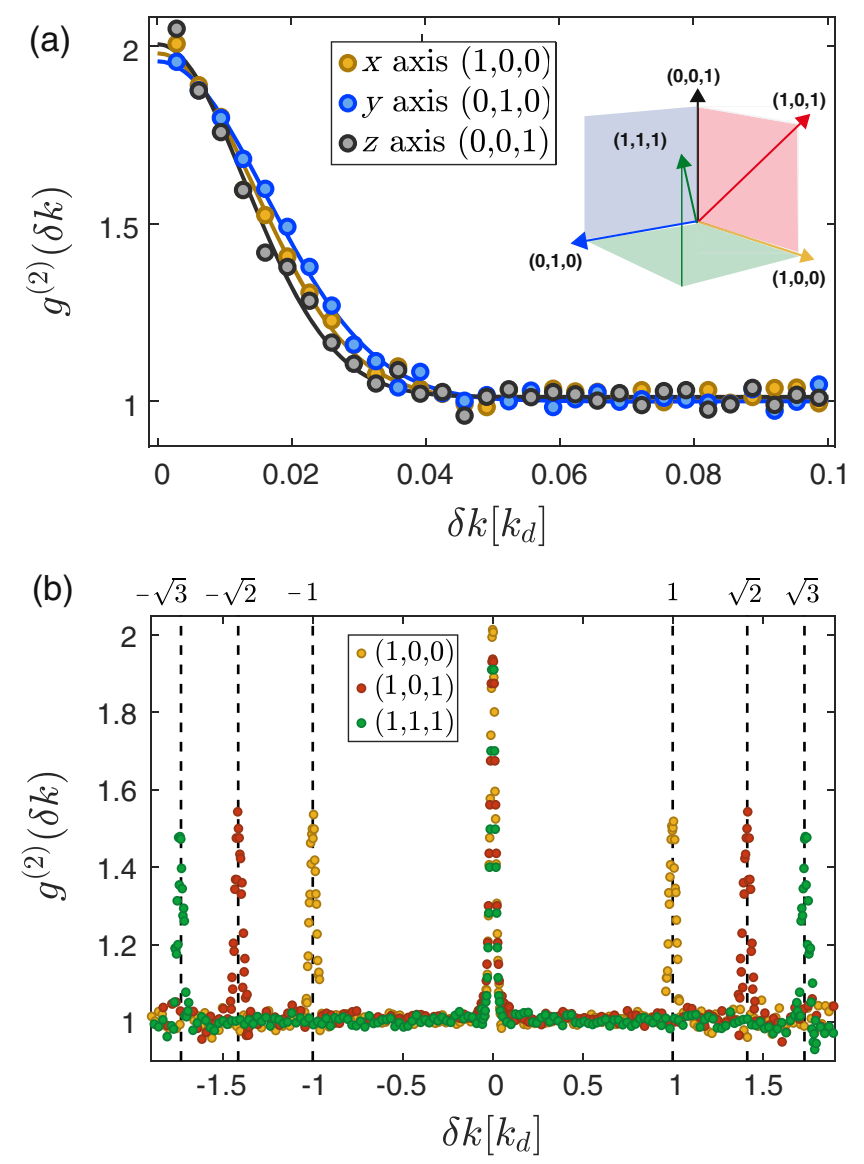

FIG. 2. Two-body momentum correlations in bosonic Mott insulators at $U / J=100$ with $N=15000$. (a) Plot of $1 \mathrm{D}$ cuts through the two-particle momentum correlation $g^{(2)}(\delta k)$ as a function of $\delta k=\delta \vec{k} \cdot \vec{u}$ along the axis defined by $\vec{u}=n_{x} \vec{u}_{x}+$ $n_{y} \vec{u}_{y}+n_{z} \vec{u}_{z}$. The values of the integers $\left(n_{x}, n_{y}, n_{z}\right)$ are indicated next to the symbols of the data. The elementary volume we use to code the positions of individual atoms has a longitudinal length $\Delta k_{\|}=0.003 k_{d}$ along the axis where correlations are plotted and a transverse length $\Delta k_{\perp}=0.015 k_{d}$. The 1D cuts are plotted along the three axes of the cubic lattice, illustrating the spherical symmetry of the experimental configuration. (b) $1 \mathrm{D}$ cuts $g^{(2)}(\delta k)$ plotted on a broader scale through the $g^{(2)}(\delta \vec{k})$ along different axes of the reciprocal lattice.

plot $g^{(2)}(\delta \vec{k})$ in a Mott insulator with $U / J=100$ along $\vec{u} \propto n_{x} \vec{u}_{x}+n_{y} \vec{u}_{y}+n_{z} \vec{u}_{z}$, where $\left\{\vec{u}_{j}\right\}_{x, y, z}$ are the orthonormal vectors associated to the lattice and $n_{x}, n_{y}$, and $n_{z}$ are equal to 0 or 1 . The two-body correlation function is plotted as a function of the difference $\delta k$ of momentum between two atoms found along the $\vec{u}$ axis (see Appendix C). We find a well-contrasted bunching whose amplitude $g^{(2)}(0)=$ $1.98(3)$ is close to two, as expected for Gaussian statistics. The corresponding large fluctuations in the momentum space are in contrast with the reduced atom-number fluctuations in the lattice sites, as monitored with in situ quantum gas microscopes in a similar regime of the Mott insulator [10]. Note that there have been only a few observations of $g^{(2)}(0) \simeq 2$ with massive particles $[35,46,47]$ and that none of them was obtained in a 3D isotropic situation. As illustrated in Fig. 2(b), the two-body correlation $g^{(2)}(\vec{k})$ is $3 \mathrm{D}$ periodic. We find periods equal to $k_{d}, \sqrt{2} k_{d}$, and $\sqrt{3} k_{d}$ along the different axes of the 3D reciprocal lattice associated with the crystalline structure of the ground state. As we explain below, the lower amplitude of the bunching peaks at $\delta \vec{k} \neq \overrightarrow{0}$ is due to small imperfections of the $\mathrm{He}^{*}$ detector.

We now turn to investigating the shape and width of the two-body correlation function. In the vicinity of $\delta \vec{k}=\overrightarrow{0}$, we find that $g^{(2)}(\delta \vec{k})$ is well fitted by an isotropic 3D Gaussian function. This isotropy corresponds to the isotropy of the optical trap created by the lattice beams. The Gaussian-like shape probably results from the spherical distribution of atoms in the lattice and from the absence of sharp edges in the in-trap density. These observations about the isotropy and the shape of the measured two-body correlations lead us to define the two-particle correlation length $l_{c}$ according to $g^{(2)}(\delta \vec{k})=1+\eta \exp \left(-2 \delta \vec{k}^{2} / l_{c}^{2}\right)$ at $|\delta \vec{k}| \ll k_{d}$, where $\eta$ corresponds to the amplitude of the bunching. We find a correlation length $l_{c}=0.027(6) k_{d}$ for the data in Fig. 2(a).

As discussed in the theory section, the two-body correlations of a perfect Mott state are known accurately, as one can compute $g^{(2)}(\delta \vec{k})=1+\left|g^{(1)}(\delta \vec{k})\right|^{2}$ from the sum appearing in Eq. (3). This computation requires the knowledge of the spatial distribution of atoms in the 3D lattice, which we obtain using the Gutzwiller ansatz for the experimental parameters [31]. To investigate in the experiment how the shape of $g^{(2)}(\delta \vec{k})$ is modified when the spatial distribution of the atoms in the 3D lattice varies, we use the low compressibility of the gas: The size $L$ of a trapped Mott insulator increases with the atom number $N$ as a result of the strong on-site interactions. We vary the atom number from $N=3.0(6) \times 10^{3}$ to $N=22(4) \times 10^{3}$ and measure the different correlation lengths $l_{c}$. Over this range, we note that the lattice filling at the trap center varies from 1 to 2 , but numerical calculations of $g^{(2)}$ show that the presence of a few doubly occupied sites at the trap center hardly affects the value of $l_{c}$. In Fig. 3, we plot the measured $l_{c}$ as a function of the atom number $N$. The two-body correlation length $l_{c}$ is found to decrease with $N$, as expected from the fact that $L$ increases with $N$. We also plot $l_{c}$ as a function of the size $L$ obtained from the Gutzwiller ansatz in the inset in Fig. 3 (see Appendix $\mathrm{C}$ for details). Moreover, the measured values for $l_{c}$ are in perfect agreement with numerical calculations without adjustable parameters, i.e., with the expectation for the atom distribution of a Mott state and for a Gaussian density operator. Note that, in a thermal gas of noninteracting bosons, the size $L$ and, in turn, $l_{c}$ are independent of $N$. The observed variation of $l_{c}$ with $N$ thus highlights the difference between probing the Mott state and probing an ideal Bose gas [35]. As a side remark, we note that an analogy with the Hanbury-Brown 


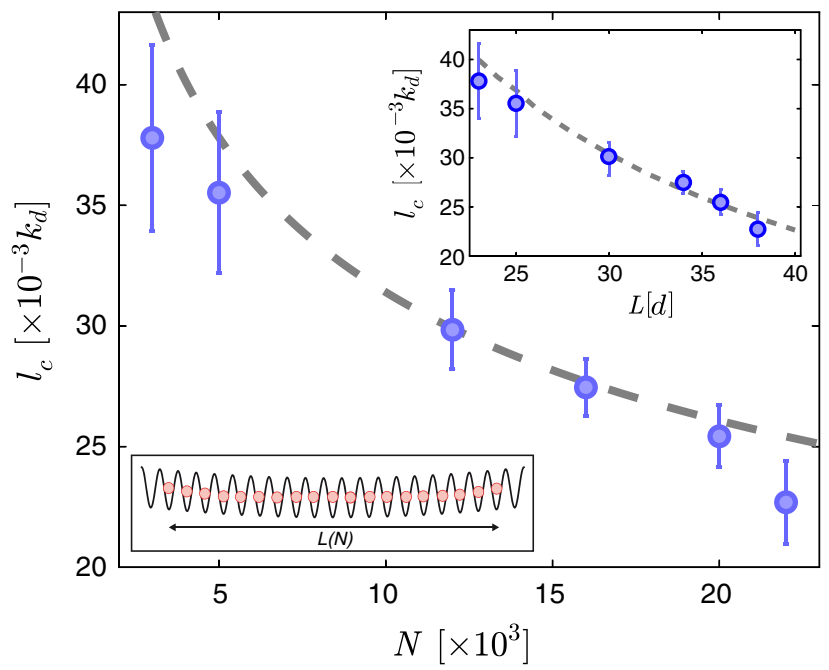

FIG. 3. Two-body correlation length. Correlation length $l_{c}$ plotted as a function of the total atom number $N$ in the Mott state. The dashed line is the theoretical prediction from 3D numerical calculations without adjustable parameters. Inset: $l_{c}$ as a function of the calculated in-trap total size $L$. The dashed line is the same numerical calculation as that in the main panel.

and Twiss effect $[21,48]$ can also be used to describe the observed two-body correlations (see Appendix A for details).

Proceeding similarly with a Gaussian fit, we measure the two-body correlation length $l_{c}^{\delta k \neq 0}=0.033(9) k_{d}$ for the peaks in Fig. 2(b) observed at $\delta \vec{k} \neq \overrightarrow{0}$. This value is slightly larger than $l_{c}$ (measured at $\delta \vec{k}=\overrightarrow{0}$ ) by a few times $k_{a} / 1000$. This tiny discrepancy may be explained by small imperfections of the $\mathrm{He}^{*}$ detector in measuring particle distances comparable to its radius (see, for instance, Refs. [49,50], and Appendix C). While the measured correlation lengths and amplitudes may be affected by the response function of the detector, the 3D integral of the bunching peak, which quantifies the probability for two atoms to bunch, should be a physical quantity conserved through the detection. In a crystalline ground state as a Mott insulator, atoms are expected to have the same probability to bunch modulo a vector $\vec{K}$ of the reciprocal lattice. It implies that the 3D integrals of the bunching peaks should be equal on the reciprocal lattice. Importantly, we find that this property is verified in the experiment (see Appendix $\mathrm{C}$ ). This result implies that the observed amplitude $g^{(2)}(\vec{K} \neq \overrightarrow{0})<2$ results from the measured correlation length $l_{c}^{\delta k \neq 0}$ larger than $l_{c}$.

From the measured 3D atom distributions, we can extract higher-order correlations. To illustrate this possibility, we measure the three-body correlations. We observe a well-contrasted and periodic bunching in the threebody correlations $g^{(3)}\left(\vec{k}, \overrightarrow{k^{\prime}}, \overrightarrow{k^{\prime \prime}}\right)$ whose numerator is written $\left\langle\hat{a}^{\dagger}(\vec{k}) \hat{a}^{\dagger}\left(\overrightarrow{k^{\prime}}\right) \hat{a}^{\dagger}\left(\overrightarrow{k^{\prime \prime}}\right) \hat{a}(\vec{k}) \hat{a}\left(\overrightarrow{k^{\prime}}\right) \hat{a}\left(\overrightarrow{k^{\prime \prime}}\right)\right\rangle$. In Fig. 4(a), we plot $g^{(3)}\left(\delta k_{1}, \delta k_{2}\right)$ as a function of $\delta k_{1}$ and $\delta k_{2}$, where $\delta k_{1}=$ $\left(\vec{k}-\vec{k}^{\prime}\right) \cdot \vec{u}_{x}$ and $\delta k_{2}=\left(\vec{k}-\vec{k}^{\prime \prime}\right) \cdot \vec{u}_{x}$ are the differences of

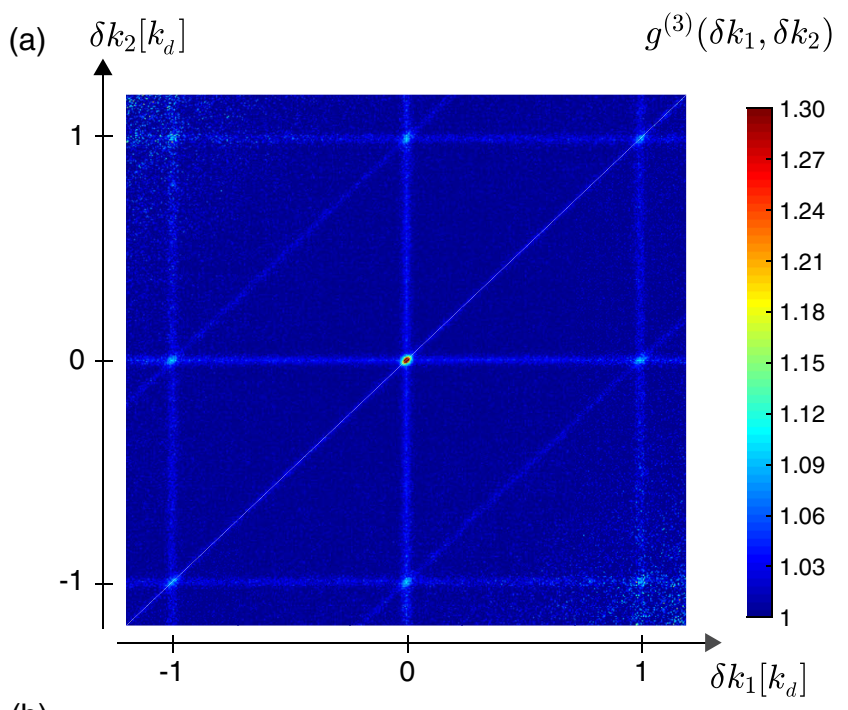

(b)

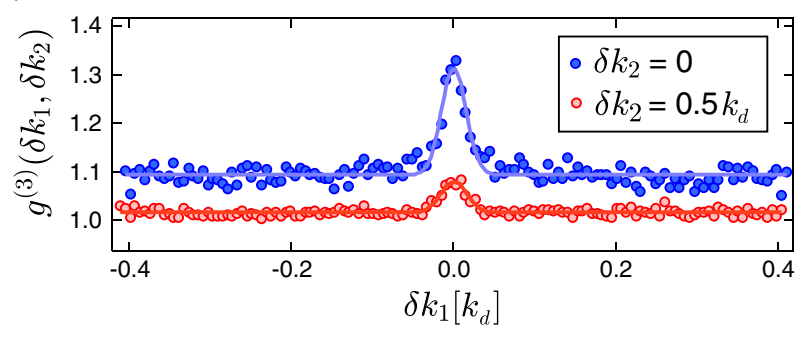

FIG. 4. Three-body momentum correlations in bosonic Mott insulators. (a) Plot of $g^{(3)}\left(\delta k_{1}, \delta k_{2}\right)$ as a function of the algebraic distances $\delta k_{1}$ and $\delta k_{2}$ between atoms along the lattice axis (see the text). The background amplitude associated to uncorrelated atoms has an amplitude of approximately 1 . The correlations along the lines defined by $\delta k_{1}=0, \delta k_{2}=0$, and $\delta k_{1}=\delta k_{2}$ correspond to the two-body bunching $g^{(2)}(0)$ associated with finding two atoms close by. The intrinsic three-body correlations (located at $\delta k_{1}=0\left[k_{d}\right], \delta k_{2}=0\left[k_{d}\right]$ ) have an even higher amplitude. Here, we use $\Delta k_{\|}=0.008 k_{d}$ and $\Delta k_{\perp}=0.1 k_{d}$. (b) Profiles of $g^{(3)}\left(\delta k_{1}, \delta k_{2}\right)$ at $\delta k_{2}=0$ and $\delta k_{2}=0.5 k_{d}$. We find $g^{(3)}(0,0)-$ $1=0.32(2)$ and $g^{(3)}\left(0,0.5 k_{d}\right)=0.065(10)$, i.e., that the ratio between the amplitudes of three-body and two-body correlations is compatible with the expected value for Gaussian statistics.

momentum between two different pairs of atoms along the lattice axis $\vec{u}_{x}$. The correlations that are intrinsically threebody are those for which three atoms are in the same coherence volume, and, thus, they are located at positions $\left(\delta k_{1}=0\left[k_{d}\right], \delta k_{2}=0\left[k_{d}\right]\right)$, where $\left[k_{d}\right]$ stands for modulo $k_{d}$. We also observe lines with a correlation above the background along the axis defined by $\delta k_{1}=0$, by $\delta k_{2}=0$, and by $\delta k_{1}=\delta k_{2}$. Along each of these lines, two of the three atoms are close to each other, and the correlation amplitude signals two-body bunching. No correlation is observed on the antidiagonal defined by $\delta k_{1}=-\delta k_{2}$. This result is expected, since it corresponds to well-separated, and, thus, uncorrelated, momentum components. Finally, the ratio of the amplitudes of the intrinsic threebody correlations $g^{(3)}(0,0)$ and the two-body ones 
(a)
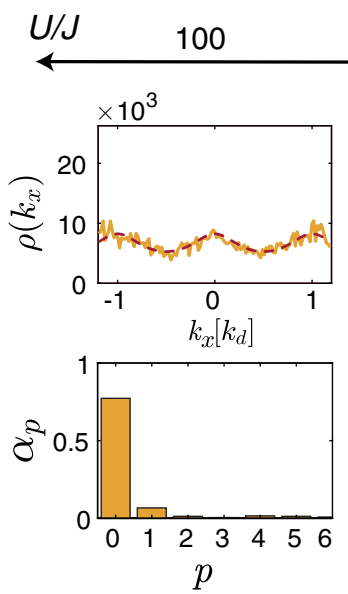

60

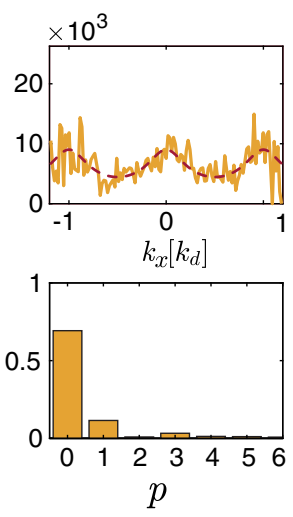

45

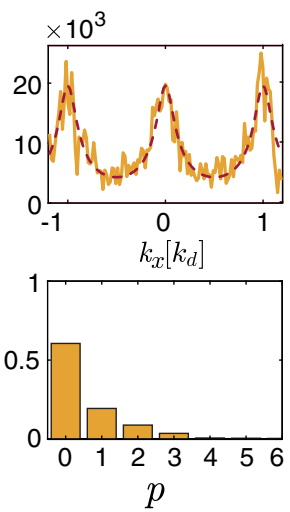

35
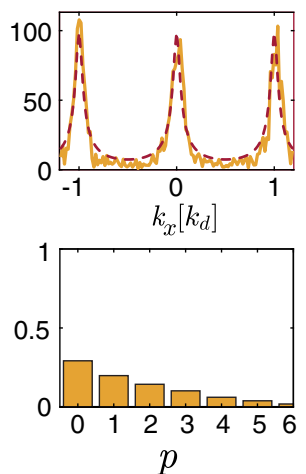

(b)

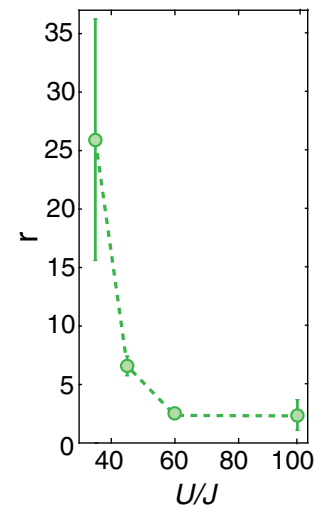

FIG. 5. Spectral weight of the quasiparticles in the Mott state. (a) Top row: Dimensionless momentum densities $\rho\left(k_{x}\right) \propto Z\left(k_{x}\right)-1$ in the Mott regime for a ratio $U / J=100,60,45,35$. The dashed line is the momentum density expected from perturbation many-body theories accounting for the presence of particle-hole excitations, with the value of $U / J$ taken as a fitting parameter [40]. Bottom row: Histograms of the amplitudes $\alpha_{p}$ corresponding to the momentum densities displayed on top. (b) Ratio $r=\left[U / J-(U / J)_{c}\right] /$ $\left[u_{\mathrm{fit}}-(U / J)_{c}\right]$ plotted as a function of $U / J$. The ratio $r$ quantifies the discrepancy between $U / J$ set in the experiment and $u_{\mathrm{fit}}=U / J$ obtained from fitting the experimental data with perturbative theories.

$g^{(3)}\left(0, \delta k_{2} \neq 0\right)$ is found to be $2.9(4)$, a value compatible with the expected one of $3 ! / 2 !=3$ for Gaussian statistics [35]. Note that this ratio is obtained for transverse integrations $\left(\Delta k_{\perp}=0.1 k_{d}\right)$ larger than those used in Fig. 2 in order to increase the statistics of the three-body correlations. As a consequence, the measured absolute amplitudes are smaller than the ones expected in the absence of integration [Fig. 4(b)].

\section{Quasiparticle spectral weight in a Mott insulator}

When $U / J$ is decreased, a structure slowly appears in the momentum density. Measuring momentum 3D densities in the far field and without the line-of-sight integration inherent to absorption imaging allows us to investigate this evolution quantitatively. In Fig. 5(a), we plot $\rho\left(k_{x}\right)$ in the Mott regime along the lattice axis $O x$ and for a varying ratio $U / J$. All these datasets are taken with a fixed atom number $N=3.0(6) \times 10^{3}$ to ensure unity occupation of the lattice at the trap center. At large $U / J$, we recover the periodic oscillations observed previously [23,24]. For decreasing $U / J$, we observe that $\rho\left(k_{x}\right)$, and, thus, the spectral weight $Z\left(k_{x}\right) \propto \rho\left(k_{x}\right)+1$, becomes increasingly peaked around $k_{x}=j k_{d}$ with $j$ an integer. To provide a physical insight into the narrowing of $Z\left(k_{x}\right)$, we expand $\rho\left(k_{x}\right)=\sum_{p} \alpha_{p} e^{i 2 \pi p k_{x} / k_{d}}$ in Fourier components along the lattice axis, finding

$$
\alpha_{p}=\sum_{j}\left\langle\hat{b}_{j}^{\dagger} \hat{b}_{j+p}\right\rangle
$$

The Fourier amplitudes $\alpha_{p}$ quantify the average phase coherence between lattice sites distant by $p$ sites. The evolution of the amplitudes $\alpha_{p}$ as $U / J$ decreases highlights the continuous change from a perfect Mott state (for which $\alpha_{p}=0$ for $\left.p \neq 0\right)$ to a Mott state with a finite spatial coherence $\left(\alpha_{p} \neq 0\right.$ for $\left.p>1\right)$. Close to the Mott transition, the mobility of the quasiparticles extends over several lattice sites, say, distant by $p$ sites, and results in $\alpha_{p} \neq 0$. The narrowing of the spectral weight $Z\left(k_{x}\right)$ thus reflects the coherent tunneling of the quasiparticles over several lattice sites. In Fig. 5(a) (bottom row), we plot the Fourier amplitudes $\alpha_{p}$ extracted from the measured profiles $\rho\left(k_{x}\right)$. A mobility of the quasiparticles over up to $p \sim 6$ lattice sites is observed at $U / J=35$, a distance corresponding to about a quarter of the size of the trapped gas.

The spectral weight of a homogeneous Mott with unity occupation of the lattice sites is calculated using perturbative many-body approaches [38-40]. It provides a quantitative prediction of the role of particle-hole excitation on the building of long-range coherence which we confront with the experiment. To do so, we fit the measured densities $\rho\left(k_{x}\right)$ with Eq. (95) of Ref. [40] using the numerical parameters associated with a 3D lattice and letting the ratio $U / J=u_{\text {fit }}$ as a fit parameter (i.e., the parameter $x$ of Ref. [40]). The observed modification in the shape of $Z\left(k_{x}\right)$ as the system approaches the superfluid regime is consistent with these theoretical predictions [see Fig. 5(a)]. As the predicted width and amplitude of the modulations are connected through the dispersion of the lattice, this agreement suggests that the physical picture drawn by perturbative theories is valid.

However, a fully quantitative comparison reveals that $u_{\text {fit }}$ is systematically smaller than the ratio $U / J$ calibrated in the experiment. To quantify the discrepancy between the theory and experiment, we define a fractional deviation as the ratio $r=\left[U / J-(U / J)_{c}\right] /\left[u_{\mathrm{fit}}-(U / J)_{c}\right]$, and we 
plot $r$ as a function $U / J$ in Fig. 5(b). We find that $r$ is large close to the critical ratio $(U / J)_{c}$ and that it decreases as the ratio $U / J$ increases, becoming closer to unity. Our understanding of this observation goes as follows. Close to the Mott transition, the trapped system realized in the experiment is composed of a Mott insulator phase surrounded by a condensate. The latter obviously increases the coherence of the trapped system with respect to that predicted by the theory. As $U / J$ increases, the Mott region with unity filling extends over a larger volume in the trap, and the outer shell becomes normal. As a result, $r$ should become closer to unity at increasing $U / J$. However, we do not observe $r=1$ at large $U / J$, but $r \simeq 2$, a difference that may be related to the increasing difficulty to adiabatically load the atoms in the lattice at increasing $U / J$. We note that the observed trend for the ratio $r$ differs from that found in a previously reported work with a similar quantity, the visibility [39]. It is because the theoretical model we use here, which captures the position of the Mott transition predicted by quantum Monte Carlo calculations, is different from that used in Ref. [39].

\section{CONCLUSION}

We have reported on the measurement of $3 \mathrm{D}$ atom distributions in momentum space for a Mott insulator. It has allowed us to determine quantitatively the buildup of long-range coherence when approaching the transition towards a superfluid. Deep in the Mott phase, the extracted two- and three-body correlation functions match that of indistinguishable and uncorrelated bosons uniformly distributed in the lattice sites. It shows that the measured correlations are that of a many-body state whose density operator is Gaussian in momentum space. The results of this work also demonstrate the outstanding capability of our approach to identify Gaussian quantum states. It naturally paves the way to the future investigation of 3D many-body states with many-body correlations deviating from those associated with Gaussian statistics. As illustrated with 1D Bose gases [51], these deviations provide genuine information about many-body correlations and have, therefore, the potential to unveil complex quantum phenomena.

\section{ACKNOWLEDGMENTS}

We acknowledge fruitful discussions with D. Boiron, F. Gerbier, T. Roscilde, and all the members of the Quantum Gases group at Institut d'Optique. We acknowledge financial support from the LabEx PALM (Grant No. ANR-10LABX-0039), the Région Ile-de-France in the framework of the DIM SIRTEQ, and, and the Agence Nationale pour la Recherche (Grant No. ANR-17-CE30-0020-01). D. C. acknowledges support from the Institut Universitaire de France.

\section{APPENDIX A: OPTICAL ANALOGY OF THE MOMENTUM-SPACE BUNCHING}

The description of the momentum-space two-body correlations we investigate in this work could be rephrased in an optical analogy. Indeed, the momentum-space properties are obtained in a TOF experiment where the matter wave evolves freely in space similarly to the propagation of light. When the expansion of the gas is ballistic and long enough for the paraxial approximation to be valid, the atoms are detected in a regime identical to that of the detection of photons in the far field. The momentum-space bunching phenomenon can thus be understood from a direct analogy with the Hanbury-Brown and Twiss (HBT) effect reported with incoherent sources of light [48] or matter [21,52].

It is also interesting to recall that the properties of the HBT effect in optics may be derived from a classical model of light with Gaussian statistics [53]. This derivation requires one to assume that a large number of mutually incoherent emitters form the source of light, a condition under which the central limit theorem applies and the field is a Gaussian random process. This assumption is a classical analog of the hypothesis of a Gaussian ground state we use here, and it highlights the fact that in both situations the HBT type of correlations are connected with Gaussian statistics.

\section{APPENDIX B: ATOM-NUMBER CALIBRATION AND HE* DETECTOR}

\section{Calibration of the atom number in the lattice}

The data presented in this work come from various sets of the experiment:

(i) A series of sets taken with $N=3.0(6) \times 10^{3}$ atoms in the lattice and for $U / J=35,45,60$, and 100.This atom number ensures a unity occupation of the lattice sites at the trap center and for all the lattice amplitudes. These sets are used for Figs. 1 and 4 and for the points at $L=23$ and $L=25$ in Fig. 3 .

(ii) A set of data at $U / J=100$ with $N=15 \times 10^{3}$ atoms, corresponding to a filling at the trap center equal to 2.-This larger atom number increases the signal-to-noise ratio to measure the two-body correlation length. We check by performing numerical simulations that the bunching peaks are quite insensitive to the presence of a few doubly occupied sites and that $l_{c}$ is dominated by the width $L$ of the trapped gas. Taking into account atom-number fluctuations in the experiment, the overall data are divided into four sets of data with $N=12(3) \times 10^{3}$, $N=16(3) \times 10^{3}, \quad N=20(4) \times 10^{3}, \quad$ and $\quad N=$ $22(4) \times 10^{3}$ atoms.

To calibrate the atom number, Mott insulators at $U / J=$ 100 are probed by absorption imaging after $1.5 \mathrm{~ms}$ TOF. At this lattice amplitude, the momentum distribution is diluted, 
and absorption images can be fitted by a 2D Gaussian function from which one can extract the number of atoms.

\section{Detection with the $\mathrm{He}^{*}$ detector}

To access single-atom-resolved momentum distributions, the atoms are detected on the $\mathrm{He}^{*}$ detector located $43 \mathrm{~cm}$ below the center of the science chamber, corresponding to a time of flight of $297 \mathrm{~ms}$. To avoid the effect of spurious magnetic fields that would disturb the time-offlight distribution, a fraction of the atoms, initially spin polarized into the $2^{3} S_{1} m_{J}=1$ state, are transferred with a radio-frequency (rf) resonant pulse to the nonmagnetic $m_{J}=0$ state at the beginning of the TOF. The atoms remaining in a state with a nonzero magnetic moment are expelled from the detection area by the use of a magnetic gradient. By adjusting the duration of the rf pulse, the fraction of atoms transferred to the nonmagnetic state, in which atoms are detected, can be controlled. To make sure that there is no saturation effect of the He* detector, we choose to detect about $5 \%$ of the atom number per shot. Between 200 and 2000 shots are then taken for each specific measurement.

\section{APPENDIX C: CORRELATION FUNCTIONS EXTRACTED FROM THE MEASURED ATOM DISTRIBUTIONS}

The measurement of two-particle momentum correlations quantifies the conditional probability for an atom in a given experimental realization to have a momentum $\vec{k}_{1}$ provided one atom is detected with a momentum $\vec{k}_{2}$ :

$$
G^{(2)}\left(\vec{k}_{1}, \vec{k}_{2}\right)=\left\langle\hat{a}^{\dagger}\left(\vec{k}_{1}\right) \hat{a}^{\dagger}\left(\vec{k}_{2}\right) \hat{a}\left(\vec{k}_{2}\right) \hat{a}\left(\vec{k}_{1}\right)\right\rangle .
$$

In our experiment, the measurement of the full 3D momentum distribution with single-atom sensitivity allows us to compute the 3D correlation functions. However, as the representation of the full distribution is intricate, we calculate two-body correlations along some specific directions $\vec{k}_{1}-\vec{k}_{2} \propto \vec{u}$. These directions can be chosen at will, and, in the present work, they are oriented along the reciprocal lattice vector $\vec{u} \propto n_{x} \vec{u}_{x}+n_{y} \vec{u}_{y}+n_{z} \vec{u}_{z}$ with $n_{x}, n_{y}$, and $n_{z}$ integers and $\vec{u}_{x}, \vec{u}_{y}$, and $\vec{u}_{z}$ the lattice axes. The translation invariance of the state we probe implies that $G^{(2)}$ depends only on the momentum separation between two particles, e.g,. $G^{(2)}\left(\vec{k}_{1}, \vec{k}_{2}\right)=G^{(2)}(\delta k \vec{u})$ with $\vec{k}_{1}-\vec{k}_{2}=\delta k \vec{u}$. We consequently calculate $G^{(2)}$ along the lattice direction by integrating over the position of one of the two atoms:

$G^{(2)}(\delta \vec{k})=\int_{\vec{k}}\left\langle\hat{a}^{\dagger}(\vec{k}) \hat{a}^{\dagger}(\vec{k}+\delta \vec{k}) \hat{a}(\vec{k}+\delta \vec{k}) \hat{a}(\vec{k})\right\rangle d \vec{k}$.

Here, the integral refers to the summation over all the atoms present in one shot of the experiment. To increase

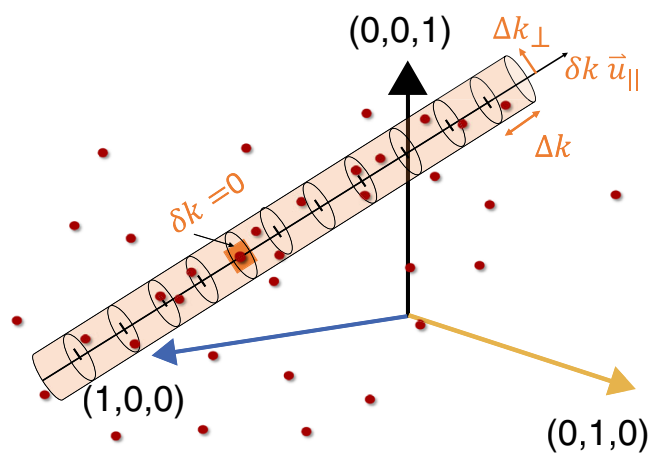

FIG. 6. Method to calculate $G^{(2)}$. One atom from a given shot is chosen (highlighted in orange). A tube of radius $\Delta k_{\perp}$ oriented along $\vec{u}$ and centered on the considered atom is defined. The distances $\delta k$ between the atom and the other ones contained in the tube are extracted. The operation is repeated for all the atoms of the shot, and the results are saved in a histogram with a longitudinal step $\Delta k$.

the signal-to-noise ratio, we also perform a transverse integration:

$$
G^{(2)}(\delta k \vec{u})=\int_{\left|\vec{k}_{\perp}\right|<\Delta k_{\perp}} G^{(2)}\left(\delta k \vec{u}+\vec{k}_{\perp}\right) d \vec{k}_{\perp} .
$$

The procedure to calculate $G^{(2)}(\delta k)$ is depicted in Fig. 6. For each atom, we define a tube of radius $\Delta k_{\perp}$ oriented along $\vec{u}$ from which the histogram of the distances from this atom to the ones contained in the tube is recorded. The histograms corresponding to the different atoms of the shots are then summed and $G^{(2)}$ averaged over many realizations of the experiment. A plot of $G^{(2)}$ measured along the $x$ axis and corresponding to the data displayed in Fig. 2 is given in Fig. 7(a). Three bunching peaks are visible at $\delta k=0$ and $\delta k= \pm k_{d}$ on top of a broad background which can be identified as resulting from the Fourier transform of the Wannier function. This background is equal to the autocorrelation of the momentum density of the Mott insulator. It corresponds to the value of the $G^{(2)}$ function in the absence of correlation between atoms and later referred as $G_{\mathrm{NC}}^{(2)}$. The ratio $G^{(2)}$ divided by $G_{\mathrm{NC}}^{(2)}$ yields the normalized two-body correlation function $g^{(2)}$ used in the main text. By definition, $g^{(2)}(\delta k)=1$ if there is no correlation in the system, and $g^{(2)}(\delta k) \neq 1$ otherwise [see Fig. 7(b)].

To ensure a proper normalization, we calculate $G_{\mathrm{NC}}^{(2)}$ with a procedure similar to that used for $G^{(2)}$ but by getting rid of the correlations before performing the calculation of the histogram. Removing the correlations is obtained from considering the atoms belonging to all the shots, since there are no correlations between two atoms belonging to two different shots of the experiment. The value of $G_{\mathrm{NC}}^{(2)}$ for the same set of data mentioned earlier is given in Fig. 7(a). 

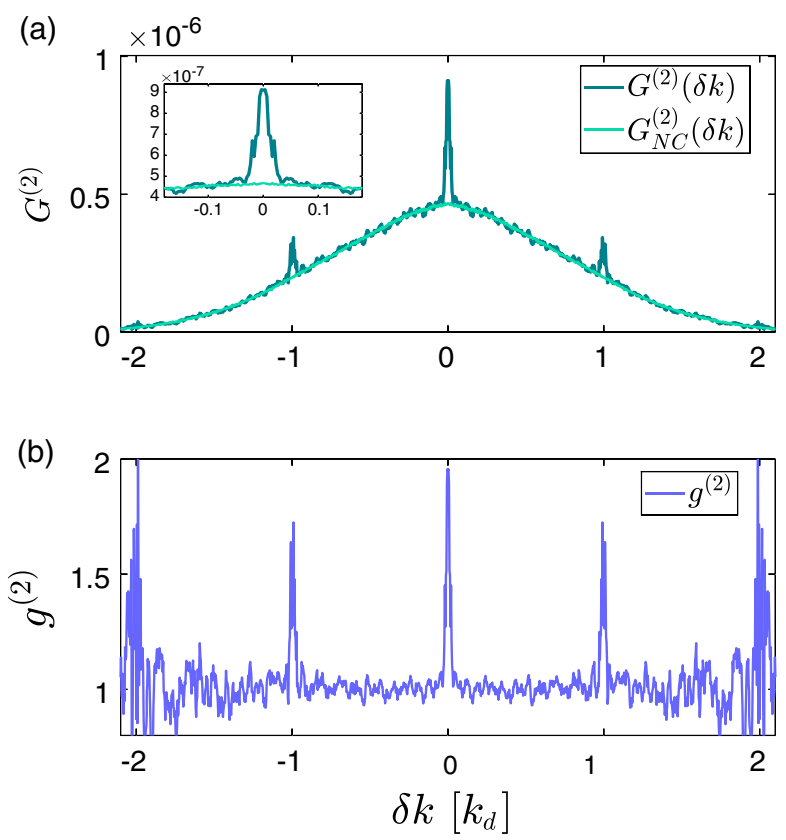

FIG. 7. Correlation functions. Value of the different correlation functions along the $x$ axis for the data displayed in Fig. 2. (a) $G^{(2)}(\delta k)$ and $G_{\mathrm{NC}}^{(2)}(\delta k)$. (b) $g^{(2)}(\delta k)$.

The result of dividing $G^{(2)}$ by $G_{\mathrm{NC}}^{(2)}$, i.e., $g^{(2)}(\delta k)=$ $G^{(2)}(\delta k) / G_{\mathrm{NC}}^{(2)}(\delta k)$, is plotted in Fig. 7(b) for the same set of data used in Fig. 7(a).

\section{Effect of transverse integration}

The volume of integration $\Delta k \times \Delta k_{\perp}^{2}$ may affect the shape of $g^{(2)}(\delta k)$ when $\Delta k, \Delta k_{\perp} \approx l_{c}$, with $l_{c}$ the correlation length of $g^{(2)}(\delta k)$ as defined in the main text. To avoid affecting the size and shape of the correlation peaks, we use $\Delta k \approx 0.1 l_{c}$. See Table I. Although the transverse integration does not modify the shape of the correlation peaks, it results in decreasing the bunching amplitude $\eta$ defined by $g^{(2)}(\delta k)=$ $1+\eta \exp \left(-2 \delta k^{2} / l_{c}^{2}\right)$. Figure 8 displays the measured value of $\eta$ as a function of $\Delta k_{\perp}$, illustrating this assertion.

For the data shown in Fig. 2, we use $\Delta k_{\perp}=0.015 k_{d}$, which is smaller than $l_{c}=0.027(4) k_{d}$. At a lower transverse integration, the signal becomes too noisy (see Fig. 8).

TABLE I. Parameters used to compute the data in Fig. 3. In order to measure the correlation length with a good precision, we use $\Delta k_{\perp} \approx l_{c}$, resulting in $\eta=g^{(2)}(\delta k=0)-1<1$.

\begin{tabular}{lcccccc}
\hline \hline$L[d]$ & $N\left[10^{3}\right]$ & $\Delta N\left[10^{3}\right]$ & $l_{c}\left[k_{d}\right]$ & $\Delta k_{\perp}\left[k_{d}\right]$ & $\Delta k_{\|}\left[k_{d}\right]$ & $\eta \ldots$ \\
\hline 23 & 3 & 1 & 0.038 & 0.03 & 0.002 & 0.38 \\
25 & 5 & 1 & 0.035 & 0.03 & 0.002 & 0.32 \\
30 & 12 & 2 & 0.03 & 0.03 & 0.001 & 0.51 \\
34 & 16 & 3 & 0.027 & 0.03 & 0.001 & 0.43 \\
36 & 20 & 4 & 0.025 & 0.03 & 0.001 & 0.42 \\
38 & 22 & 4 & 0.023 & 0.03 & 0.001 & 0.40 \\
\hline \hline
\end{tabular}
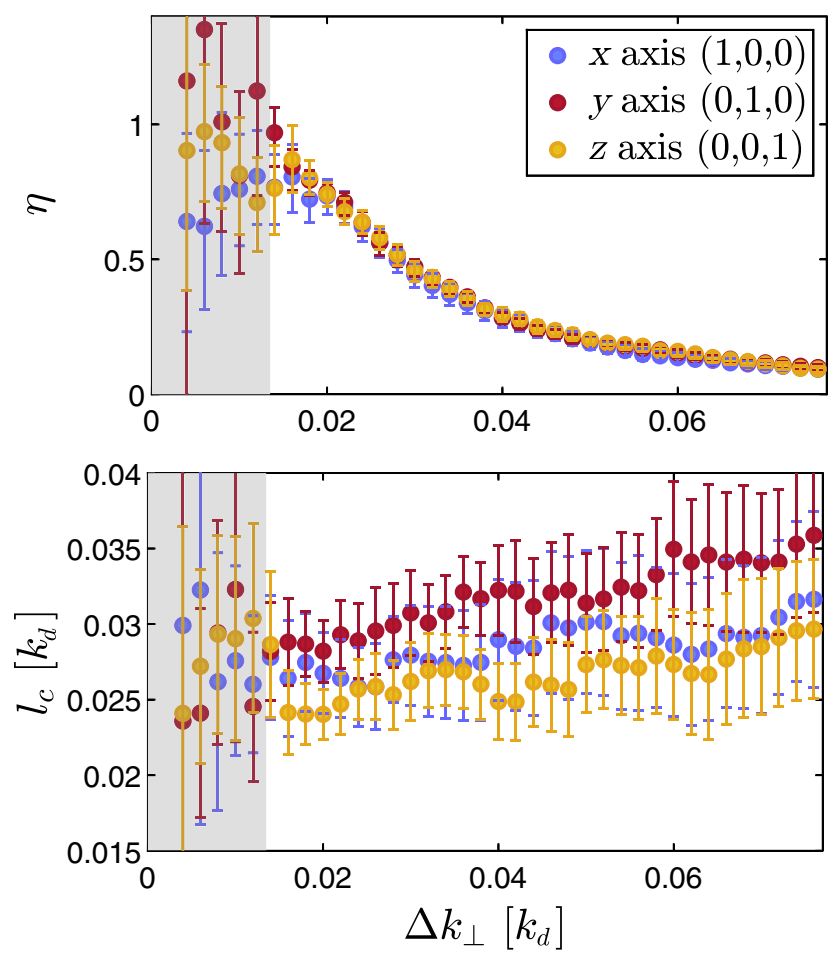

FIG. 8. Effect of the transverse integration on $g^{(2)}(\delta k)$. (a) Amplitude of the bunching $\eta$ as a function of $\Delta k_{\perp}$ for the data used in Fig. 2. (b) Correlation length $l_{c}$ as a function of $\Delta k_{\perp}$. Gray areas on both graphs correspond to the region where the signal is too noisy to provide quantitative information.

To further increase the signal-to-noise ratio in order to reach a precise measurement of $l_{c}$, we use $\Delta k_{\perp} \approx l_{c}$ in Fig. 3. Consequently, the bunching amplitude $\eta$ lies between 0.3 and 0.5 (see Table I).

\section{Numerical calculation of the correlation length $\boldsymbol{l}_{c}$}

The dashed line in Fig. 3 is obtained numerically with the following procedure. First, we calculate the three-dimensional distribution of atoms from the Gutzwiller ansatz, which is valid at large $U / J$. Second, we assume that atoms in the different lattice sites are fully decoupled. The resulting density operator being Gaussian, we can use the Wick theorem to show that $g^{(2)}(\delta k)=$ $1+\left|g^{(1)}(\delta k)\right|^{2}$ and, thus, evaluate numerically $g^{(2)}(\delta k)$ from summing the contributions of all the atoms to the one-body correlation $g^{(1)}$. This procedure is identical to that used in Ref. [21].

\section{3D integral of the bunching peaks on the reciprocal lattice}

As explained in the main text, we find an identical 3D integral for the bunching peaks associated with the reciprocal lattice. Table II contains the measured amplitudes and correlation lengths of the different peaks in $g^{(2)}$ shown in Fig. 2(b). We also give the values of the ratio between the 3D 
TABLE II. Results from fitting the bunching peaks in $g^{(2)}(\delta k)$ with a Gaussian. $\eta$ and $l_{c}$ are, respectively, the amplitude and the correlation length of the bunching at $\delta k=0 . \eta^{\neq 0}$ and $l_{c}^{\neq 0}$ are, respectively, the amplitude and the correlation length of the bunching at $\delta k \neq 0$. The ratio of the 3D integral of the bunching peaks at $\delta k=0$ and at $\delta k \neq 0$ is close to 1 along any direction of the reciprocal lattice.

\begin{tabular}{llll}
\hline \hline Direction & {$[1,0,0]$} & {$[1,1,0]$} & {$[1,1,1]$} \\
\hline$\eta$ & $0.98(2)$ & $0.94(3)$ & $1.0(2)$ \\
$l_{c}$ & $0.029(1)$ & $0.031(1)$ & $0.026(1)$ \\
$\eta^{\neq 0}$ & $0.51(1)$ & $0.49(1)$ & $0.45(2)$ \\
$l_{c}^{\delta k \neq 0}$ & $0.035(1)$ & $0.037(2)$ & $0.033(1)$ \\
$\left(\eta / \eta^{\neq 0}\right)\left(l_{c} / l_{c}^{\neq 0}\right)^{3}$ & $1.04(6)$ & $1.15(8)$ & $1.12(8)$ \\
\hline \hline
\end{tabular}

integrals of the peak located at $\delta k=0$ and that located at the position $\delta k \neq 0$ of the reciprocal lattice, $\left(\eta l_{c}^{3}\right) /\left[\eta^{\neq 0}\left(l_{c}^{\neq 0}\right)^{3}\right]$.

As the $3 \mathrm{D}$ integral of the bunching peak is found constant at any $\delta \vec{k}=\vec{K}$, the lower amplitude in $g^{(2)}(\delta \vec{k})$ observed at $\delta \vec{k}=\vec{K} \neq \overrightarrow{0}$ results from the measured larger correlation length. We stress that the measured increase in the correlation length amounts only to a few times $k_{a} / 1000$. The capability to measure such a small difference demonstrates the outstanding performances of the $\mathrm{He}^{*}$ detector. On the other hand, it is most probable that the origin of this tiny discrepancy lies in small imperfections of the $\mathrm{He}^{*}$ detector.

More specifically, a detector made of microchannel plates (MCPs) and a delay-line anode - as in the $\mathrm{He}^{*}$ detector-is better at measuring small distances than large ones between individual particles. Small distortions in the image of a regular pattern are indeed reported on distances comparable with the MCP diameter [49]. In addition, a recent investigation with $\mathrm{MeV} \alpha$ particles also shows that the uncertainty on the measure of distances of the order of the MCP diameter may be up to 4 times larger than that on the measure of small distances [50]. To our knowledge, a full understanding of these distortions is missing, but several origins for these imperfections have been identified. It includes the inhomogeneity of the electric field on the edges of the MCP and the presence of mechanical imperfections in the delay-line anode-e.g., an imperfect winding of the cables realizing the electronic waveguide.

In our experiment, the resolution with which the position of individual atoms is reconstructed is estimated to be $\sigma \sim 2.5 \times 10^{-3} k_{d}$. The increase in the correlation length $l_{c}^{\neq 0} \simeq 1.2 l_{c}$ is compatible with an accuracy to measure large particle separation which is 3 times worse (approximately $7.5 \times 10^{-3} k_{d}$ ).

[1] J. I. Cirac and P. Zoller, Goals and Opportunities in Quantum Simulation, Nat. Phys. 8, 264 (2012).

[2] G. D. Mahan, Many-Particle Physics (Springer, New York, 2000).
[3] A. Damascelli, Z. Hussain, and Z. X. Shen, Angle-Resolved Photoemission Studies of the Cuprate Superconductors, Rev. Mod. Phys. 75, 473 (2003).

[4] J. M. Tranquada, B. J. Sternlieb, J. D. Axe, Y. Nakamura, and S. Uchida, Evidence for Stripe Correlations of Spins and Holes in Copper Oxide Superconductors, Nature (London) 375, 561 (1995).

[5] M. Imada, A. Fujimori, and Y. Tokura, Metal-Insulator Transitions, Rev. Mod. Phys. 70, 1039 (1998).

[6] G. R. Stewart, Heavy-Fermion Systems, Rev. Mod. Phys. 56, 755 (1984).

[7] P. A. Lee, N. Nagaosa, and X.-G. Wen, Doping a Mott Insulator: Physics of High-Temperature Superconductivity, Rev. Mod. Phys. 78, 17 (2006).

[8] H. Ott, Single Atom Detection in Ultracold Quantum Gases: A Review of Current Progress, Rep. Prog. Phys. 79, 054401 (2016).

[9] N. F. Mott, The Basis of the Electron Theory of Metals, with Special Reference to the Transition Metals, Proc. Phys. Soc. (London) Sect. A 62, 416 (1949).

[10] J. F. Sherson, C. Weitenberg, M. Endres, M. Cheneau, I. Bloch, and S. Kuhr, Single-Atom-Resolved Fluorescence Imaging of an Atomic Mott Insulator, Nature (London) 467, 68 (2010).

[11] W. S. Bakr, A. Peng, M. E. Tai, R. Ma, J. Simon, J. I. Gillen, S. Folling, L. Pollet, and M. Greiner, Probing the Superfluid-to-Mott Insulator Transition at the Single Atom Level, Science 329, 547 (2010).

[12] M. Endres, M. Cheneau, T. Fukuhara, C. Weitenberg, P. Schauss, C. Gross, L. Mazza, M. C. Banuls, L. Pollet, I. Bloch, and S. Kuhr, Observation of Correlated ParticleHole Pairs and String Order in Low-Dimensional Mott Insulators, Science 334, 200 (2011).

[13] R. Ma, M. Tai, P. Preiss, W. Bakr, J. Simon, and M. Greiner, Photon-Assisted Tunneling in a Biased Strongly Correlated Bose Gas, Phys. Rev. Lett. 107, 095301 (2011).

[14] M. Cheneau, P. Barmettler, D. Poletti, M. Endres, P. Schau, T. Fukuhara, C. Gross, I. Bloch, C. Kollath, and S. Kuhr, Light-Cone-Like Spreading of Correlations in a Quantum Many-Body System, Nature (London) 481, 484 (2012).

[15] A. Mazurenko, C. S. Chiu, G. Ji, M. F. Parsons, M. KanaszNagy, R. Schmidt, F. Grusdt, E. Demler, D. Greif, and M. Greiner, A Cold-Atom Fermi-Hubbard Antiferromagnet, Nature (London) 545, 462 (2017).

[16] T. Hilker, G. Salomon, F. Grusdt, A. Omran, M. Boll, E. Demler, I. Bloch, and C. Gross, Revealing Hidden Antiferromagnetic Correlations in Doped Hubbard Chains via String Correlators, Science 357, 484 (2017).

[17] T. Stoferle, H. Moritz, C. Schori, M. Kohl, and T. Esslinger, Transition from a Strongly Interacting $1 D$ Superfluid to a Mott Insulator, Phys. Rev. Lett. 92, 130403 (2004).

[18] D. Clément, N. Fabbri, L. Fallani, C. Fort, and M. Inguscio, Exploring Correlated 1D Bose Gases from the Superfluid to the Mott-Insulator State by Inelastic Light Scattering, Phys. Rev. Lett. 102, 155301 (2009).

[19] N. Fabbri, S. Huber, D. Clément, L. Fallani, C. Fort, M. Inguscio, and E. Altman, Quasiparticle Dynamics in a Bose 
Insulator Probed by Interband Bragg Spectroscopy, Phys. Rev. Lett. 109, 055301 (2012).

[20] E. Altman, E. Demler, and M. D. Lukin, Probing ManyBody States of Ultracold Atoms via Noise Correlations, Phys. Rev. A 70, 013603 (2004).

[21] S. Folling, F. Gerbier, A. Widera, O. Mandel, T. Gericke, and I. Bloch, Spatial Quantum Noise Interferometry in Expanding Ultracold Atom Clouds, Nature (London) 434, 481 (2005).

[22] T. Rom, T. Best, D. van Oosten, U. Schnieder, S. Folling, B. Paredes, and I. Bloch, Free Fermion Antibunching in a Degenerate Atomic Fermi Gas Released from an Optical Lattice, Nature (London) 444, 733 (2006).

[23] F. Gerbier, A. Widera, S. Folling, O. Mandel, T. Gericke, and I. Bloch, Phase Coherence of an Atomic Mott Insulator, Phys. Rev. Lett. 95, 050404 (2005).

[24] I. B. Spielman, W. D. Phillips, and J. V. Porto, Mott-Insulator Transition in a Two-Dimensional Atomic Bose Gas, Phys. Rev. Lett. 98, 080404 (2007).

[25] R. Bücker, A. Perrin, S. Manz, T. Betz, C. Koller, T. Plisson, J. Rottmann, T. Schumm, and J. Schmiedmayer, Single-Particle-Sensitive Imaging of Freely Propagating Ultracold Atoms, New J. Phys. 11, 103039 (2009).

[26] B. Fang, A. Johnson, T. Roscilde, and I. Bouchoule, Momentum-Space Correlations of a One-Dimensional Bose Gas, Phys. Rev. Lett. 116, 050402 (2016).

[27] P. M. Preiss, J. H. Becher, R. Klemt, V. Klinkhamer, A. Bergschneider, and S. Jochim, High-Contrast Interference of Ultracold Fermions, arXiv:1811.12939.

[28] S. S. Hodgman, R. I. Khakimov, R. J. Lewis-Swan, A. G. Truscott, and K. V. Kheruntsyan, Solving the Quantum Many-Body Problem via Correlations Measured with a Momentum Microscope, Phys. Rev. Lett. 118, 240402 (2017).

[29] H. Cayla, C. Carcy, Q. Bouton, R. Chang, G. Carleo, M. Mancini, and D. Clément, Single-Atom-Resolved Probing of Lattice Gases in Momentum Space, Phys. Rev. A 97, 061609(R) (2018).

[30] M. Greiner, O. Mandel, T. Esslinger, T. W. Hansch, and I. Bloch, Quantum Phase Transition from a Superfluid to a Mott Insulator in a Gas of Ultracold Atoms, Nature (London) 415, 39 (2002).

[31] D. Jaksch, C. Bruder, J. I. Cirac, C. W. Gardiner, and P. Zoller, Cold Bosonic Atoms in Optical Lattices, Phys. Rev. Lett. 81, 3108 (1998).

[32] B. Capogrosso-Sansone, N. V. Prokof'ev, and B. V. Svistunov, Phase Diagram and Thermodynamics of the ThreeDimensional Bose-Hubbard Model, Phys. Rev. B 75, 134302 (2007).

[33] M. Born and E. Wolf, Principles of Optics: Electromagnetic Theory of Propagation, Interference and Diffraction of Light, 6th ed. (Pergamon, Oxford, 1993), Chap. 10.

[34] G. W. Gardiner and P. Zoller, Quantum Noise (SpringerVerlag, Berlin, 2000).

[35] R. G. Dall, A. G. Manning, S. S. Hodgman, W. Rugway, K. V. Kheruntsyan, and A. G. Truscott, Ideal n-Body
Correlations with Massive Particles, Nat. Phys. 9, 341 (2013).

[36] D. van Oosten, P. van der Straten, and H. T. C. Stoof, Quantum Phases in an Optical Lattice, Phys. Rev. A 63, 053601 (2001).

[37] E. Altman and A. Auerbach, Oscillating Superfluidity of Bosons in Optical Lattices, Phys. Rev. Lett. 89, 250404 (2002).

[38] K. Sengupta and N. Dupuis, Mott-Insulator-to-Superfluid Transition in the Bose-Hubbard Model: A Strong-Coupling Approach, Phys. Rev. A 71, 033629 (2005).

[39] F. Gerbier, A. Widera, S. Folling, O. Mandel, T. Gericke, and I. Bloch, Interference Pattern and Visibility of a Mott Insulator, Phys. Rev. A 72, 053606 (2005).

[40] J. K. Freericks, H. R. Krishnamurthy, Y. Kato, N. Kawashima, and N. Trivedi, Strong-Coupling Expansion for the Momentum Distribution of the Bose-Hubbard Model with Benchmarking against Exact Numerical Results, Phys. Rev. A 79, 053631 (2009).

[41] M. Iskin and J. K. Freericks, Superfluidity of p-Wave and s-Wave Atomic Fermi Gases in Optical Lattices, Phys. Rev. A 80, 063610 (2009).

[42] Q. Bouton, Q. Bouton, R. Chang, A. L. Hoendervanger, F. Nogrette, A. Aspect, C. I. Westbrook, and D. Clément, Fast Production of Bose-Einstein Condensates of Metastable Helium, Phys. Rev. A 91, 061402(R) (2015).

[43] F. Nogrette, D. Heurteau, R. Chang, Q. Bouton, C. I. Westbrook, R. Sellem, and D. Clément, Characterization of a Detector Chain Using a FPGA-Based Time-to-Digital Converter to Reconstruct the Three-Dimensional Coordinates of Single Particles at High Flux, Rev. Sci. Instrum. 86, 113105 (2015).

[44] F. Gerbier, S. Trotzky, S. Folling, U. Schnorrberger, J. D. Thompson, A. Widera, I. Bloch, L. Pollet, M. Troyer, B. Capogrosso-Sansone, N. V. Prokofev, and B. V. Svistunov, Expansion of a Quantum Gas Released from an Optical Lattice, Phys. Rev. Lett. 101, 155303 (2008).

[45] I. Bloch, J. Dalibard, and S. Nascimbene, Quantum Simulations with Ultracold Quantum Gases, Nat. Phys. 8, 267 (2012).

[46] A. Ottl, S. Ritter, M. Kohl, and T. Esslinger, Correlations and Counting Statistics of an Atom Laser, Phys. Rev. Lett. 95, 090404 (2005).

[47] R. Lopes, A. Imanaliev, M. Bonneau, J. Ruaudel, M. Cheneau, D. Boiron, and C. I. Westbrook, Second-Order Coherence of Superradiance from a Bose-Einstein Condensate, Phys. Rev. A 90, 013615 (2014).

[48] R. Hanbury-Brown and R. Q. Twiss, Correlations between Photons in Two Coherent Beams of Light, Nature (London) 177, 27 (1956).

[49] O. Jagutzki, J. Lapington, L. Worth, U. Spillman, V. Mergel, and H. Schmidt-Bocking, Position Sensitive Anodes for MCP Read-Out Using Induced Charge Measurement, Nucl. Instrum. Methods Phys. Res., Sect. A 477, 1 (2002).

[50] R. Hong, A. Leredde, Y. Bagdasarova, X. Flchard, A. Garca, P. Mller, A. Knecht, E. Linard, M. Kossin, M. G. Sternberg, H. E. Swanson, and D. W. Zumwalta, High Accuracy Position Response Calibration Method for a Micro-Channel 
Plate Ion Detector, Nucl. Instrum. Methods Phys. Res., Sect. A 835, 42 (2016).

[51] T. Schweigler, V. Kasper, S. Erne, I. Mazets, B. Rauer, F. Cataldini, T. Langen, T. Gasenzer, J. Berges, and J. Schmiedmayer, Experimental Characterization of a Quantum Many-Body System via Higher-Order Correlations, Nature (London) 545, 323 (2017).
[52] M. Schellekens, R. Hoppeler, A. Perrin, J. V. Gomes, D. Boiron, A. Aspect, and C. I. Westbrook, Hanbury-Brown Twiss Effect for Ultracold Quantum Gases, Science $\mathbf{3 1 0}$, 648 (2005).

[53] J. W. Goodman, Statistical Optics, Wiley classics library (Wiley, New York, 1985). 